\title{
L'expression de la violence dans la broderie contemporaine
}

Suivre le fil rouge de l'histoire des femmes

The expression of violence in contemporary embroidery. Following the red thread of the history of women

\section{Léonie Lauvaux}

\section{(2) OpenEdition Journals}

Édition électronique

URL : http://journals.openedition.org/cel/7816

DOI : $10.4000 /$ cel.7816

ISSN : 2262-208X

\section{Éditeur}

École du Louvre

\section{Référence électronique}

Léonie Lauvaux, "L'expression de la violence dans la broderie contemporaine », Les Cahiers de l'École du Louvre [En ligne], 15 | 2020, mis en ligne le 31 octobre 2020, consulté le 10 décembre 2020. URL http://journals.openedition.org/cel/7816 ; DOI : https://doi.org/10.4000/cel.7816

Ce document a été généré automatiquement le 10 décembre 2020.

\section{(c) (i) (9)}

Les Cahiers de l'École du Louvre sont mis à disposition selon les termes de la licence Creative Commons Attribution - Pas d'Utilisation Commerciale - Pas de Modification 4.0 International. 


\title{
L'expression de la violence dans la broderie contemporaine
}

\author{
Suivre le fil rouge de l'histoire des femmes \\ The expression of violence in contemporary embroidery. Following the red \\ thread of the history of women
}

\section{Léonie Lauvaux}

« Rouge est la couleur du sang. Rouge est la couleur de la douleur. Rouge est la couleur de la violence. Rouge est la couleur du danger. Rouge est la couleur de la honte. Rouge est la couleur de

la jalousie. Rouge est la couleur des reproches.

Rouge est la couleur des ressentiments. »

Louise Bourgeois

1 Longtemps reléguée au rang de pratique occupationnelle, la broderie est de plus en plus plébiscitée par les artistes. Quittant son caractère décoratif, elle est qualifiée de " subversive " par Rozsika Parker (The subversive stitch : embroidery and the making of the feminine, 1984) lorsqu'elle est mise au service d'un discours féminin dénonçant la domination masculine.

2 À l'image de Marie-France Dubromel, «Mercière Ambulante ${ }^{1}$ », brodant minutieusement la citation de Louise Bourgeois, le fil rouge s'illustre dans un corpus d'œuvres brodées d'artistes contemporaines. Dans un mouvement inverse que celui décrit par Colette Cosnier dans son ouvrage Le silence des filles. De la plume à l'aiguille, les plasticiennes quittent la plume pour l'aiguille et écrivent, sur le tissu tendu sur le cercle à broder, une histoire des femmes.

3 Pourquoi la broderie, pratique pensée comme féminine, devient-elle un moyen d'expression d'une violence systémique à l'égard des femmes? Quelle est la place symbolique de la couleur rouge dans ces pratiques artistiques? Comment peuvent-elles participer à l'émergence d'un matrimoine?

4 Fig. 1. 


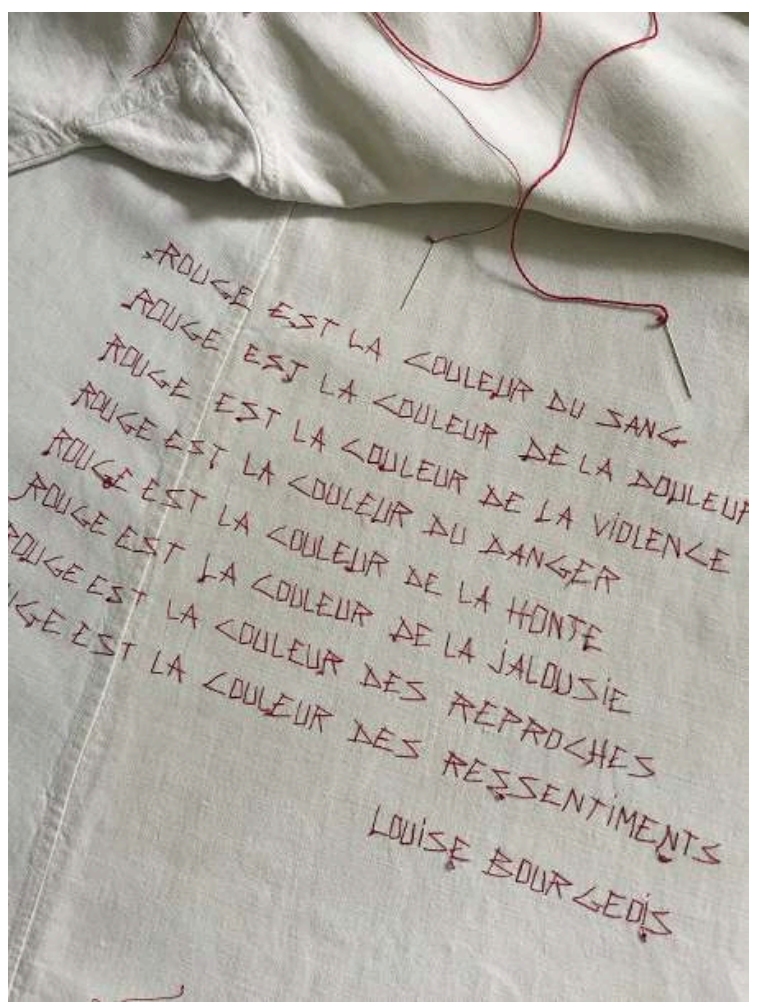

Marie-France Dubromel, Tribute to Louise Bourgeois, 2002, broderie au fil rouge sur tissu ancien, dimensions variables.

\section{Apprentissage d'un « devenir-femme »}

\section{Une vision essentialiste des femmes et du tissu}

Dans notre culture, le textile et la mercerie sont généralement considérés comme féminin(e)s. Les femmes seraient naturellement prédisposées au maniement des aiguilles, développeraient dès leur plus jeune âge un goût pour les tissus, vêtements, et autres pratiques textiles. Rousseau dans Émile ou de l'éducation écrit des fillettes qu'elles éprouvent une "répugnance à lire et à écrire; mais quant à tenir l'aiguille c'est ce qu'elles apprennent toujours volontiers. » Il conclut que cet apprentissage les plonge dans l'attente d'être femme et qu'elles songent « avec plaisir que ces talents pourront un jour leur servir à se parer $^{2}$.» Avec Au bonheur des dames, Zola va plus loin en proposant une analogie entre le plaisir éprouvé par les femmes au contact et à la vue du tissu et le plaisir sexuel. Il écrit :

«D'ailleurs, ces dames n'avaient pas lâché les dentelles. Elles s'en grisaient. Les pièces se déroulaient, allaient et revenaient de l'une à l'autre, les rapprochant encore, les liant de fils légers. C'était, sur leurs genoux, la caresse d'un tissu miraculeux de finesse, où leurs mains coupables s'attardaient. »

Puis cela va crescendo :

«L'odeur des gants de saxe, cette odeur de fauve comme sucrée de musc, la troublait d'habitude; et elle en riait parfois, elle confessait son goût pour ce parfum équivoque, où il y a de la bête en folie, tombée dans la boîte à poudre de riz d'une fille. »

Jusqu'à la jouissance : 
«Le comptoir débordait, elle plongeait les mains dans ce flot montant de guipures, de marines, de valenciennes, de chantilly, les doigts tremblants de désir, le visage peu à peu chauffé d'une joie sensuelle ; tandis que Blanche, près d'elle, travaillée de la même passion, était très pâle, la chair soufflée et molle. »

Dans ces extraits, le tissu a le rôle d'éveiller à la sensualité. La broderie, par sa capacité à mimer la pénétration par la piqûre de l'aiguille et du va-et-vient sur un tissu tendu sur tambour, redoublerai le lien sensuel qu'entretiennent les femmes avec le tissu.

8 L'idée reçue selon laquelle le textile serait féminin s'avère être liée à des dimensions sociologiques, économiques et religieuses mais a permis de pérenniser un enfermement domestique et social des femmes. De plus, la déconstruction de cet essentialisme a tant tardé à être théorisée qu'il s'est ancré dans les mentalités. Pourtant, la broderie n'a pas toujours eu ce statut de pratique occupationnelle et ce n'est qu'au Moyen Âge qu'une hiérarchisation entre les arts fait de la broderie l'apanage des femmes d'autant plus qu'elles sont exclues des ateliers des peintres.

9 Ainsi, jusque dans les années 1960, les valeurs attendues chez les jeunes filles accomplies (docilité, douceur, patience...) étaient diffusées par la religion et la littérature puis renforcées par un apprentissage obligatoire de la broderie. La marquette, pièce rassemblant tous les points de broderie ainsi que l'alphabet et les chiffres, est brodée de fil rouge. Ce fil rouge prend une valeur symbolique et va guider la vie des femmes, de l'enfance jusqu'à l'âge adulte. Ainsi, suivre le fil rouge revient à suivre l'histoire des femmes, car comme l'écrit Anne Monjaret:

«Le parcours initiatique de la fille, de l'enfance à l'état marital, ne tient qu'à un fil rouge ; il suffit de le suivre : «marquette » de la jeune écolière, initiales brodées sur le trousseau de la future mariée, sous-vêtements souillés par les menstrues, draps tachés du sang de l'hymen puis du sang de la naissance. Le destin des filles se lit dans le marquage symbolique, physiologique et matériel du linge, ce linge qui détient ainsi la mémoire du corps féminin ${ }^{3}$.»

\section{Art et féminisme}

10 Dans les années 1970, les artistes féministes font le choix d'utiliser le textile à des fins de militantisme. Par l'histoire des femmes qu'il véhicule, il est lourd d'une charge politique mise au service d'un discours sur les droits des femmes et la domination masculine dans la société et les institutions artistiques. Le textile change ainsi de statut et passe de l'univers de l'apprentissage obligatoire à celui d'une pratique plastique privilégiée. Il s'agit de dénoncer la misogynie et l'oppression par un outil qui a longtemps pérennisé la soumission et l'enfermement des femmes.

11 Néanmoins, Aline Dallier-Popper met en garde les plasticiennes contre le risque de s'enfermer dans ce médium et les invite à oser explorer les autres formes artistiques traditionnelles. Le textile, facile d'accès, peu cher, inscrit dans une histoire des femmes, permet l'émergence d'un discours féministe mais il peut, en même temps, enfermer les artistes-femmes dans une catégorie artistique pensée comme mineure, n'appartenant pas à la catégorie des Beaux-Arts. "Ainsi, elles peuvent être artisanes, mais non artistes; elles peuvent faire, même bien faire, mais pas créer ${ }^{4} . »$ La légitimité du médium textile est à prouver en même temps qu'il se développe et Aline Dallier-Popper témoin de l'émergence de ces nouvelles pratiques, va les théoriser dans sa thèse, Activités et réalisations de femmes dans l'art. Un premier exemple: les œuvres dérivées des techniques textiles traditionnelles, soutenue en 1980. 

artistes et deviennent le véhicule de revendications féministes de la troisième vague portant sur ce que Camille Froidevaux-Metterie nomme le «tournant génital de la lutte et de la pensée féministe ${ }^{5}$ ». Le féminisme de la seconde vague avait porté davantage son attention sur l'accès à la contraception et à l'avortement permettant aux femmes de sortir d'un devoir de maternité tandis qu'aujourd'hui, les femmes revendiquent la réappropriation de leur intimité, de leur corps, de leurs désirs. Ces revendications se sont heurtées à des problèmes internes aux féminismes car travailler sur les spécificités des corps et des vécus féminins sans tomber dans le différentialisme s'avère être un travail délicat.

\section{Sois belle et tais-toi}

Traditionnellement, toutes les jeunes filles françaises avaient l'obligation d'apprendre à marquer. Seules quelques privilégiées pouvaient approfondir leurs connaissances de la "broderie blanche». Cette broderie fine et décorative servant à broder le drap "de dessus » avait vocation à être vue, illustrant la pureté de la jeune brodeuse, tandis que la broderie rouge des «draps du dessous " rappelait à la femme sa condition d'être impur. Marlène Albert-Llorca, professeure émérite en anthropologie, insiste ${ }^{6}$ sur la distinction essentielle entre deux types de travaux d'aiguille, le marquage, exécutée au fil rouge et la broderie, exécutée au fil blanc. Elle explique :

«Marquer consiste à broder son trousseau et ses draps "du dessous". Mais ce terme renvoie également aux menstrues : les marquer, c'est tenter de les réguler afin d'en contenir leur impuretéf.» choix de la couleur rouge de la marquette n'est donc pas un choix anodin. De plus, dans la symbolique des couleurs, le rouge est considéré comme une couleur ambivalente contenant en elle tous les antagonismes: amour et violence, force et faiblesse, noblesse et souillure, beauté des lèvres rouges de Blanche-Neige et duplicité $\mathrm{du}$ diable. Mais surtout, le rouge est la couleur du sang et comme le brode Fanny Viollet $^{8}$, "le sang marque». Cette petite phrase brodée de rouge fait partie d'un ensemble plus grand, Des lettres brodées, pièce réalisée à l'occasion de sa soutenance de maîtrise en arts plastiques en 1982. Pour sa réalisation, elle brode à la manière d'un abécédaire classique, invoquant ainsi la tradition du marquage du linge et des draps du dessous, non seulement brodés de rouge, mais souillés de sang. Ainsi le rouge redouble de sens, il est la marque à la fois matérielle et symbolique du féminin.

Fig. 2. 


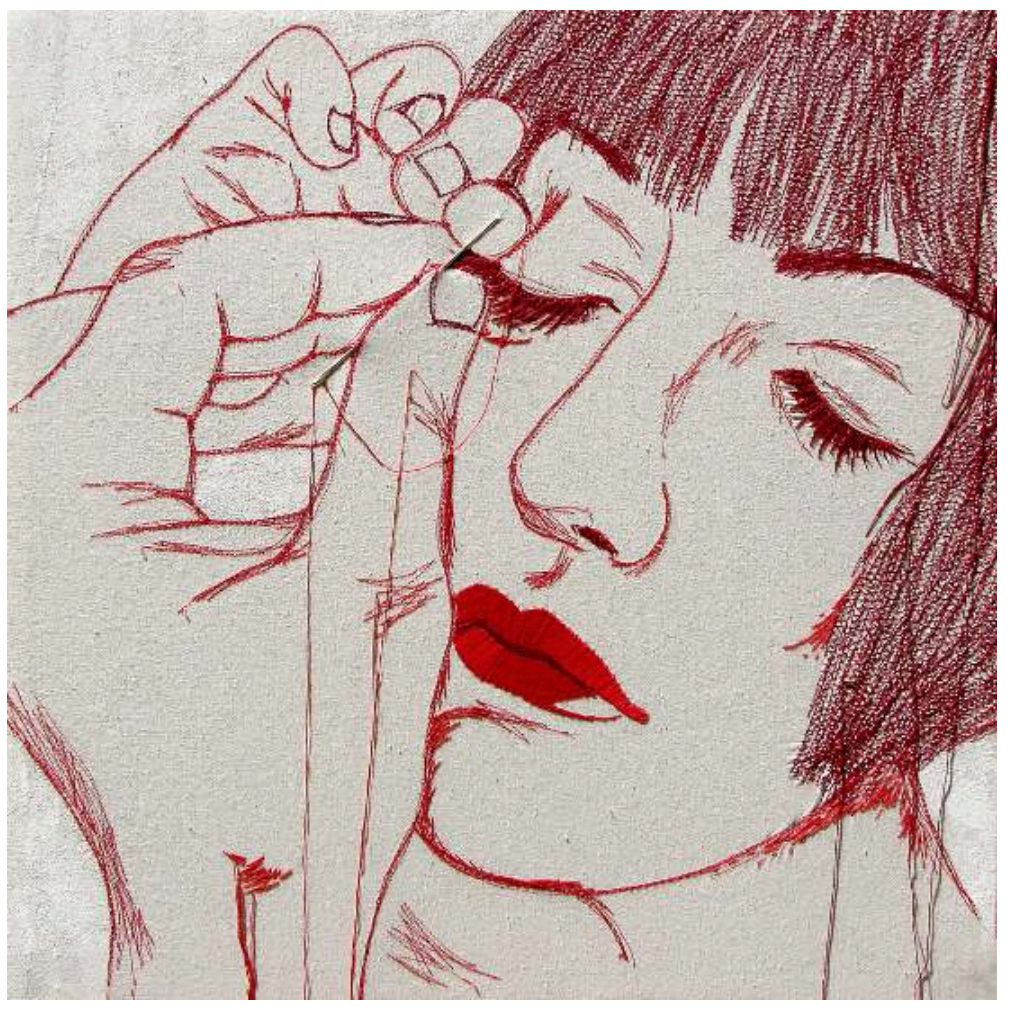

Ilaria Margutti, Mend faces V, 2008-2009, broderie sur coton, $50 \times 50 \mathrm{~cm}$. of $m e^{10}$, se compose de cinq tableaux carrés, représentant chacun un acte de broderie mimant ceux du maquillage. Derrière un geste a priori quotidien, celui de s'embellir, l'artiste met en lumière une violence banalisée faite à l'image des femmes.

La série ne présente que des gros plans du visage, de face ou de trois quarts, dont les contours sont brodés de rouge. On peut y voir une femme tenant une aiguille entre son pouce et son index, tirant sur sa paupière gauche comme pour en rehausser la ligne des sourcils. La reprise, acte courant de couture, permet à la brodeuse de repriser une paupière à grands coups de fil rouge, laissant entrevoir le passage du fil dans la chair. Alors que la broderie-maquillage est pensée comme une possibilité fragile et temporaire de s'embellir, de répondre à des canons de beauté, d'entretenir le mythe de la féminité, certaines poses prises par la femme-brodeuse-brodée, déforment son visage et ses mains, la rendant ainsi monstrueuse. Les lèvres entièrement brodées signent la fin de la séance de torture "quotidienne ", le fil rouge marque la peau d'un maquillage indélébile. Dans l'intimité de la salle de bain ou face au miroir se trame une mascarade, celle de la féminité. En effet Ilaria Margutti questionne l'identité féminine par l'utilisation de deux apanages de la féminité, le maquillage et la broderie. Par cette dernière, elle interroge également le statut de « femme-artiste » et se broder soi-même, renvoie à se faire soi-même, seule, en dehors des obligations sociales. Mais, pour atteindre cette potentialité émancipatrice, il faut souffrir. L'artiste illustre parfaitement l'adage affirmant qu'«il faut souffrir pour être belle», mettant en lumière un des paradoxes auxquels les femmes se plient chaque jour : se maquiller pour plaire et se plaire, tout en ayant conscience de répondre à une pression sociale les maintenant dans un rôle d'objet où l'apparence sert à l'établissement de leur valeur. 
Laura Mulvey dans son essai Visual Pleasure and Narrative Cinema ${ }^{11}$ théorise sous le terme de male gaze le regard cinématographique qu'elle qualifie de masculin, cisgenre et hétérosexuel. Le male gaze met en place un rapport de passivité et d'objectivation des femmes, regardées, observées, scrutées, pour le plaisir du regard masculin. Paradoxalement, il semblerait que les femmes cherchent ce regard, ressentant le besoin d'être désirées, c'est en tout cas ce qu'avance Pierre Bourdieu dans La domination masculine. En effet :

«La domination masculine, qui constitue les femmes en objets symboliques, dont l'être (esse) est un être-perçu (percipi), a pour effet de les placer dans un état permanent d'insécurité corporelle ou, mieux, de dépendance symbolique: elles existent d'abord par et pour le regard des autres, c'est-à-dire en tant qu'objets accueillants, attrayants, disponibles. On attend d'elles qu'elles soient " féminines ", c'est-à-dire souriantes, sympathiques, attentionnées, soumises, discrètes, retenues, voire effacées. Et la prétendue «féminité » n'est souvent pas autre chose qu'une forme de complaisance à l'égard des attentes masculines, réelles ou supposées, notamment en matière d'agrandissement à l'ego. En conséquence, le rapport de dépendance à l'égard des autres (et pas seulement des hommes) tend à devenir constitutif de leur être ${ }^{12}$."

Ainsi, l'identité féminine, soumise à la fragmentation et au désir de reconnaissance, ne pourrait se construire qu'au travers de ce regard déformant.

Fig. 3.

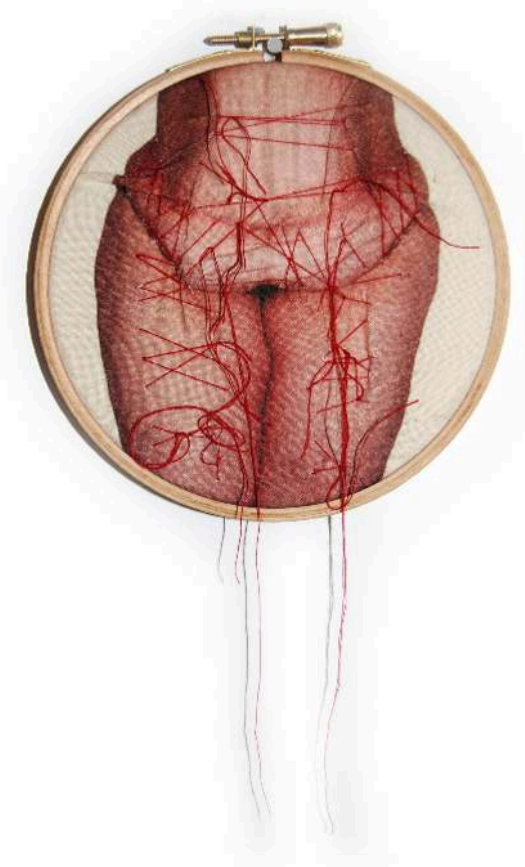

Rebecca Harris, Untilted (body modification), 2013, dimensions variables, cercle à broder, tissu imprimé et fil.

\section{(c) Rebecca Harris}

Rebecca Harris est une artiste britannique née en 1977. Pendant ses années de Master en art, elle choisit de s'engager dans une opération chirurgicale qui va influer sur ses problématiques artistiques. Étudiante obèse, elle décide de se faire opérer de l'estomac 
afin de stopper sa prise de poids. Depuis, le corps gros l'obsède, ce qu'elle traduit par l'utilisation de matériaux mous, de fils, d'objets domestiques, afin de créer des corps imaginaires, des corps hybrides, des objets sans utilités. La série «Obscure Objets of Obesity " dont est issue l'œuvre Untilted (body modification) ${ }^{13}$ se place dans l'axe de recherche "Skin as Repository : my deflated body as axis for research» La peau et le corps, à la fois dégonflés et fragmentés, sont pensés comme la mémoire d'une identité et d'un vécu.

Tout comme Ilaria Margutti, Rebecca Harris ${ }^{14}$ modèle son corps par la broderie. Les broderies sur photographies sont pour l'artiste une manière de prendre le contrôle de son corps, de le manipuler, de le changer, de le modifier, de contrer son obésité. Pour l'œuvre Untilted (body modification), Rebecca Harris a pris en photographie son corps en se focalisant sur son ventre, de la taille à mi-cuisses. Les chairs sont lourdes et forment des plis et face à ces images, l'artiste se lance dans une manipulation qu'elle ne peut effectuer sur son corps physique. En effet, à l'aide de fil rouge, elle le raye, le barre, le rature, elle pique dans la chair, tend des fils comme pour le resserrer.

Le champ lexical de la douleur et de la souffrance font référence à l'opération chirurgicale qu'a subi l'artiste, et le fil et l'aiguille sont tout autant celui de la couturière qui a pour travail de "faire la femme ", que celui du/de la chirurgien.ne qui est chargée d'ouvrir, de couper, de coudre, de refermer. Ces actes offrent une redéfinition des contours des corps, la possibilité d'une nouvelle corporalité.

Traditionnellement, broder permettait d'apprendre à se comporter en tant que future femme. Lorsque la jeune fille se piquait avec l'aiguille et qu'une goutte de sang perlait, l'expression consacrée était alors de dire que « le travail rentre ». Plus une jeune fille se piquait, plus elle apprenait les travaux d'aiguille et donc à devenir femme. Dans cette œuvre, le travail rentre, mais directement dans la chair, à même la peau. Le corps devient alors le lieu du "devenir-femme » et de l'identité féminine. Cette question de l'identité féminine est doublée par un medium "féminin » qui se revendique comme tel. Le fil rouge fait, dans les deux sens du terme, la femme.

Chez les deux artistes, la peau devient le territoire d'une mémoire où s'inscrivent littéralement les traces de la domination. Ainsi que l'écrit Pierre Bourdieu :

« Rappeler les traces que la domination imprime durablement dans les corps et les effets qu'elle exerce à travers elles, ce n'est pas apporter des armes à cette manière, particulièrement vicieuse, de ratifier la domination qui consiste à assigner aux femmes la responsabilité de leur propre oppression, en suggérant, comme on le fait parfois, qu'elles choisissent d'adopter des pratiques soumises («les femmes sont leurs pires ennemies ») ou même qu'elles aiment leur propre domination, qu'elles "jouissent» des traitements qui leur sont infligés, par une sorte de masochisme constitutif de leur nature ${ }^{15}$.»

Fig. 4. 


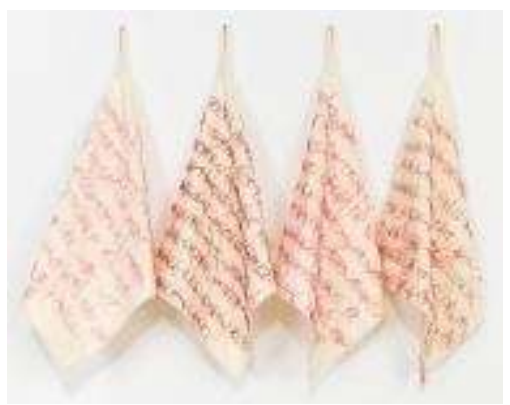

Ghada Amer, Conseil de beauté du mois d'août, 1993, broderie sur tissu, $35 \times 35 \mathrm{~cm}$, Courtesy of the artist and Marianne Boesky Gallery, New York and Aspen.

\section{(c) Ghada Amer}

Néanmoins, l'artiste Ghada Amer ${ }^{16}$ nous offre avec la série Conseil de beauté du mois d'aoû $t^{17}$ une possibilité de sortir de cette soumission. L'œuvre est composée de carrés de tissus blancs brodés en lettres capitales et en différentes nuances de rose et de rouge des conseils de beauté issus de magazines féminins. Lorsqu'ils sont accrochés dans l'espace muséal, ces morceaux de tissus tombent comme des torchons. Le terme de «torchon " qualifie tout autant le magazine dont sont issus les conseils que le torchon de cuisine de la ménagère à qui ils sont destinés. Dans ses broderies, Ghada Amer prend le parti de ne pas rentrer les fils. De ce fait, ils pendent sur l'endroit de l'ouvrage et rendent les conseils difficiles à lire, donc à suivre. Il existe un écart toujours plus grand entre un féminin idéalisé, imposé par ces conseils et la réalité de la vie féminine, rattrapée par les tâches domestiques. Ghada Amer les brode pour montrer l'impossibilité et le refus de se conformer à ces conseils. Elle les raye, elle dénonce la soumission des femmes aux obligations domestiques et aux conseils de beauté. Broder ces injonctions permet de les nier violemment, de dire non, de dire l'absurde en le brodant d'une écriture illisible et d'en renforcer l'illisibilité par l'accrochage. Il s'agit de répondre à la violence par la violence avec des armes « féminines » : le fil et l'aiguille.

1 La vie des femmes est guidée par le fil rouge, symbole du patriarcat qui les maintient dans un rôle prédéfini. Mais alors qu'il véhicule une certaine idée essentialiste de la féminité, ces broderies nous montrent que c'est aussi par lui que peut venir la libération. Il faut s'emparer du fil et de l'aiguille pour écrire sa propre histoire et sa propre idée de l'identité féminine. Selon Claude Fauque, la broderie est « héritage et renaissance. Elle relie insidieusement les femmes à une histoire, même si le fait n'est pas toujours énoncé; elle les relie aussi implicitement à l'avenir dans lequel leur ouvrage subsistera ${ }^{18}$.»

Le fil rouge relie les femmes, car comme le notait Louise Bourgeois dans son journal intime, le rouge est la couleur du sang, de la douleur, de la violence, du danger, de la honte, de la jalousie, des reproches, des ressentiments.

\section{L'écriture d'un matrimoine}

Pour un nombre non négligeable d'artistes, les pratiques textiles vont remplacer la plume et les mots, elles vont leur permettre de broder le non-dit, la violence vécue par les corps féminins : le viol, l'inceste, la naissance...

Fig. 5. 


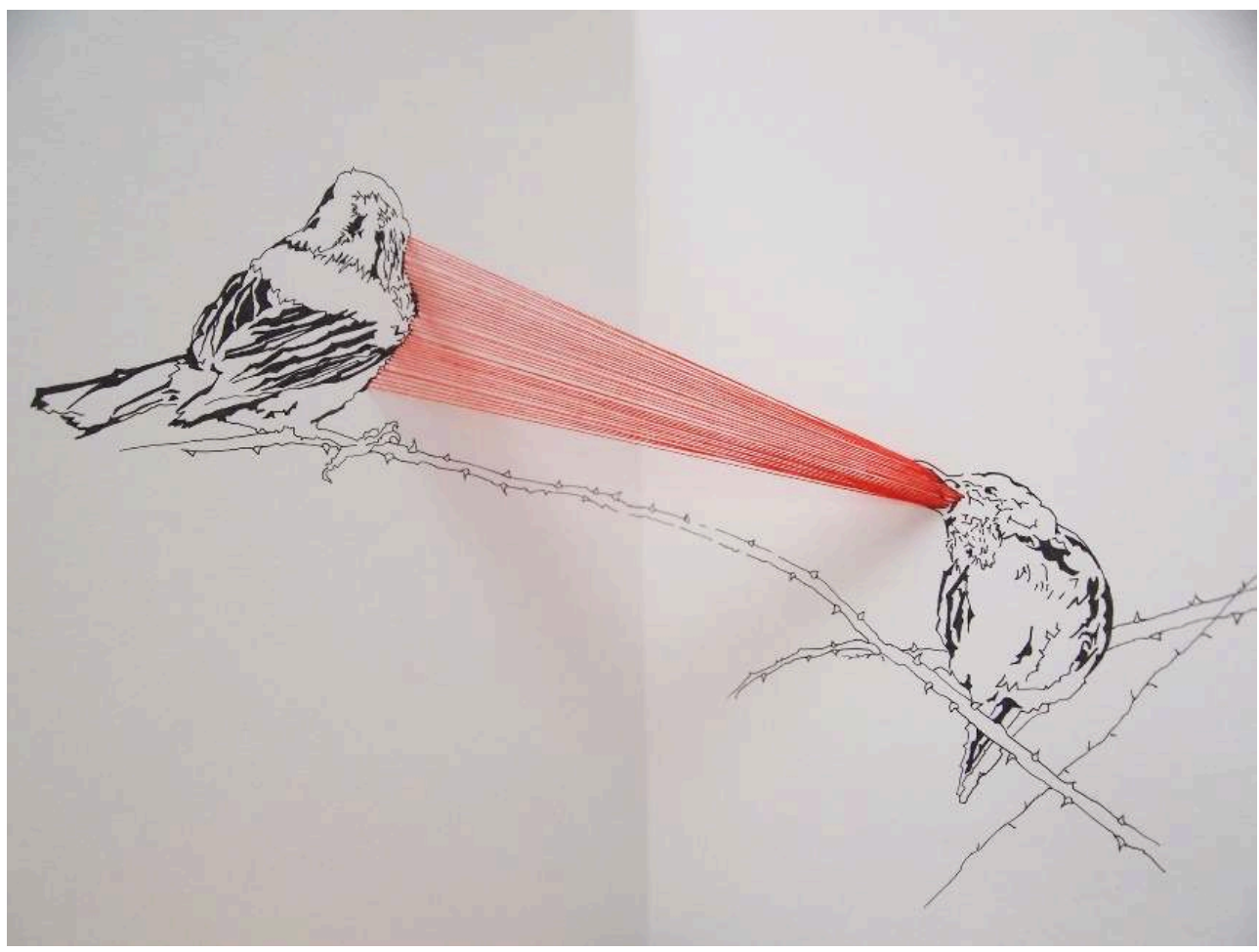

Valérie Vaubourg, Procnée et Philomèle, 2015, cadre d'angle, encre et fil tendu, galerie Anne Perré.

\section{(C) Valérie Vaubourg}

Le mythe de Philomèle et Procné issu des Métamorphoses d'Ovide est la démonstration de ce lien qu'entretiennent la broderie et les femmes. Philomèle et Procné sont toutes deux les filles de Pandion. Procné épouse Térée, roi de Thrace, avec qui elle a un fils, Itys. Sous prétexte que sa femme s'ennuie, Térée se rend à Athènes demander l'autorisation à Pandion de ramener Philomèle auprès d'elle. En chemin, il la viole puis lui coupe la langue afin qu'elle ne puisse raconter la violence subie, puis il l'enferme. Térée raconte à Procné que sa sœur est morte pendant le voyage. Philomèle, en incapacité de parler mais bien résignée à se venger, entreprend de tisser son viol. Une fois l'ouvrage terminé, une servante est chargée de le porter à Procné. Cette dernière délivre sa sœur et pour punir Térée, elles décident de tuer Itys puis de le cuire avant de le servir à manger à son père. Après le repas, il demande à voir son fils. Procné lui affirme qu'il est déjà avec lui. Devant la colère de Térée, Philomèle et Procné tentent de s'enfuir aidées par les dieux qui les transforment en hirondelle et en rossignol.

Dans ce mythe, Philomèle subit une violente ablation de deux parties de son anatomie féminine, l'hymen et la langue. La perte de l'hymen et la perte de la langue sont corrélées: la perte de la virginité signifie la perte de la parole. Pour se venger, Philomèle, Procné et la servante vont agir ensemble, sororalement. Elles tuent le fils de Térée, symbole de sa virilité et le lui font manger mettant ainsi fin à sa descendance. La mère tue son fils pour venger sa sœur, elle empêche ainsi le fils de devenir homme et de perpétuer une violence héritée de son père. Face à son incapacité à prendre la parole, puisqu'elle lui est désormais interdite, Philomèle la remplace par le fil et l'aiguille, faisant du fil un langage symboliquement féminin. En s'échappant, les deux sœurs sont transformées en oiseaux connus pour leur chant mélodieux. Grâce à sa métamorphose, Philomèle peut chanter de sa belle voix de concert avec sa sœur, renouant ainsi avec le langage. 

une part non négligeable au fil rouge. Rouge comme l'hymen perdu. Rouge comme la langue coupée. Deux oiseaux sont dessinés à l'encre noire de part et d'autre d'un coin de mur de l'espace d'exposition, sur deux différentes branches épineuses. Il s'agit de Philomèle et Procné reliées par une multitude de fils rouges tendus entre elles. Sortant $\mathrm{du}$ bec de l'une pour se prolonger jusqu'au bec et à la gorge de l'autre, les fils matérialisent la parole. Un dialogue s'établit entre les deux oiseaux, un langage féminin s'établit, une histoire féminine s'écrit. pages de papier sont remplacées par des pages de toile brodée. Cet "ouvrage" commence comme un abécédaire classique, les lettres « $\mathrm{A} »$, , $\mathrm{B}$ », « $\mathrm{C} »$, , $\mathrm{D}$ », « $\mathrm{E}$ » sont brodées de fil rouge au point de marque, selon différentes typographies. Elle écrit: «broder c'est penser avec ses mains pour donner un peu plus qu'à lire ", en fils bleus, verts et marrons. Elle donne en effet plus qu'à lire car au dos, les fils forment un arbre. Ainsi, Fanny Viollet fait de la broderie une écriture où le fil rouge fait référence à la marquette, au rouge des initiales du trousseau, au rouge du sang de l'hymen et du sang des menstrues. La broderie est un medium propre à écrire une histoire féminine, d'ailleurs, comme l'écrit Madeleine Blondel, «l'abécédaire ne serait-il pas ce journal intime où s'exprime le moi des fillettes ${ }^{22}$ ?» de Tracey $\operatorname{Emin}^{24}$ comme une prise de parole permettant la mise en place du procès fictif de son violeur. Elle donne ainsi la parole au juge, au procureur, à la victime, à l'agresseur...

41 Tracey Emin est née en 1963 d'une mère anglaise et d'un père chypriote. Ce dernier menait une double vie et était déjà marié à la naissance de l'artiste et de son frère jumeau. Ils habitaient dans l'hôtel qu'il tenait, l'« Hotel International», où le père restait quelques jours avant de passer le reste du temps avec son autre famille. L'artiste raconte sa vie à l'hôtel dans une broderie éponyme en 1993. À treize ans, le soir du nouvel an, elle est violée par un membre de son entourage proche. Après cet événement, elle rentre dans une période où elle multiplie les aventures d'un soir. Deux avortements marquent également sa vie alors qu'elle poursuit des études au Royal College of Art.

En 1995, elle réalise une tente intitulée Everyone I have Ever Slept With 1963-199525 où elle répertorie toutes les personnes avec qui elle a partagé son lit depuis 1963, l'année de sa naissance. Elle y inscrit minutieusement en broderie appliquée sur les parois d'une tente igloo bleue deux cents noms dont son frère jumeau, ses amants, sa grand-mère et ses fœtus avortés. Lorsqu'elle est ouverte, la tente semble inviter la spectatrice et le spectateur à partager l'intimité de l'artiste qui «s'ouvre » aux autres, au monde qui l'entoure. La tente est un abri précaire, nomade et partageable mais elle est aussi un lieu mystique chez les Amérindiens, utérine, elle protège et enferme à la fois. La fragilité du matériau utilisé pourrait renvoyer à la fragilité émotionnelle de l'artiste qui livre dans cette alcôve son histoire intime. Cette intimité n'est pas uniquement sexuelle, ce n'est pas un simple décompte de ses conquêtes, elle ne catégorise pas les relations entretenues, mais les range sous une seule et même catégorie : l'intimité du lit. Néanmoins, cette intimité offerte demande un engagement de la part $\mathrm{du} / \mathrm{de}$ la spectateur/trice, celui de se courber, se baisser, regarder et aller voir. Ainsi, Tracey Emin fait de nous des spectatrices et des spectateurs indiscret(e)s.

Les Cahiers de l'École du Louvre, 15 | 2020 

où l'aiguille et le tissu vont remplacer la plume et le journal intime. Ainsi, Tracey Emin reprend en quelque sorte la tradition des «diaries", journaux souvent écrits par les femmes, permettant une introspection intérieure par la narration de la quotidienneté. Tandis que les « diaries » n'ont pas vocation à être publiés, Tracey Emin fait le choix de nous donner le sien à lire. Les différentes broderies agissent comme plusieurs tomes d'un même récit, offrent une vision fragmentée à la fois violente et touchante de sa vie, bousculent les limites entre la sphère privée et la sphère publique, créant à la fois rejet et compassion envers l'artiste et sa vie. Livrer son intimité au public va devenir pour les artistes un acte artistique subversif qui va atteindre un climax dans les années 1970. Cette extimité va mener à une redéfinition de l'art et de la place de la création féminine où le corps et l'expérience sont au centre des préoccupations.

\section{Conclusion}

La broderie, à la fois héritage et matrimoine, permet l'émergence d'un discours féminin dans une société où les femmes sont exclues du pouvoir discursif. Cette exclusion du discours dominant explique leur recours à des médiums pensés comme "féminins " afin d'énoncer leur propre discours. L'histoire féminine, hors du discours dominant, est donc une histoire de l'intime. Selon Aline Dallier-Popper :

«Si l'on admet un possible rapport entre couture et écriture, l'une et l'autre assumant une fonction économique et communicative, avec, en plus, des débordements dans lesquels circulent la jouissance et la perversion, il ne parait pas si simple de survaloriser les travaux d'aiguille ni de jeter sur eux l'anathème. Situés entre assujettissement et révolte, les travaux d'aiguille pourraient fournir aujourd'hui aux femmes qui s'y consacrent, l'occasion d'analyser leur oppression et leur refoulement sexuel, et les conduire [...] à la création ${ }^{26}$. »

Ainsi, les artistes jouent avec la vision essentialiste du textile. Il devient un prétexte à la création, porteur de sens, d'une démarche et d'une mise en place d'un discours féminin et féministe œuvrant à la visibilité des femmes dans la société et les institutions. La broderie participe à l'émergence de cette écriture d'une histoire des femmes; d'ailleurs, les abécédaires traditionnels sont signés et datés et ont vocation à rester dans la famille. Se transmettant de mère en fille, ils écrivent l'histoire familiale.

La broderie participe donc à la mise en place d'un discours féminin, sur les femmes. Elle donne la parole aux femmes, écrit l'histoire des femmes, devient un exutoire à des problématiques «féminines » tues ou réprimées: la violence sexuelle et l'oppression mais aussi l'amour et le deuil, autant de situations qui jalonnent la vie des femmes.

Pour asseoir pleinement la légitimité de ce discours, les pratiques textiles contemporaines doivent être reconnues artistiquement. Ainsi, les artistes, les théoricien(ne)s et les historien(ne)s œuvrent à la réhabilitation des artistes oubliées de l'histoire de l'art officiel et mettent en place un matrimoine, permettant aux artistes contemporaines de se situer dans cette généalogie.

Néanmoins, le plafond de verre reste une réalité pour beaucoup d'artistes, qui plus est lorsqu'elles font le choix d'utiliser un medium émergeant. Cette mise à l'écart des institutions, cette mise en lumière des inégalités, cette violence systémique vont être dénoncées par l'usage d'une violence symbolique envers les femmes à grands coups d'aiguille et de fil rouge car : 
« Rouge est la couleur du sang. Rouge est la couleur de la douleur. Rouge est la couleur de la violence. Rouge est la couleur du danger. Rouge est la couleur de la honte. Rouge est la couleur de la jalousie. Rouge est la couleur des reproches. Rouge est la couleur des ressentiments. »

\section{NOTES}

1. "Marie-France Dubromel, "mercière ambulante", déambule avec son baluchon, rempli de menus objets de rebut, de chiffons, de bouts de fils, de boutons trouvés, de papiers froissées ou déchirés, d'images, de livres, d'écriture, de photographies, de reliques diverses... portant la marque d'un vécu disparu. » La mercerie ambulante (http://mercerieambulante.typepad.com/ [site consulté le 23/07/2020]).

2. Jean-Jacques Rousseau, Émile ou De l'éducation, Paris, Flammarion (réed.), 1966, p. 479.

3. Anne Monjaret, "Point de croix ", Point de croix : au bonheur des filles, Paris, Éd. de la Réunion des Musées Nationaux, 2001, p. 63.

4. Agnese Fidecaro et Stéphanie Lachat, Profession: créatrice, la place des femmes dans le champ artistique, Lausanne, Antipodes, Coll. Existence et société, 2007, p. 16.

5. Camille Froidevaux-Metterie, Le corps des femmes : la bataille de l'intime, Philosophie magazine Éditeur, 2018.

6. Marlène Albert-Llorca, « Les fils de la Vierge. Broderie et dentelle dans l'éducation des jeunes filles », Homme, 1995, n 133, pp. 99-122.

7. Idem, Ibidem.

8. Fanny Viollet est une artiste française vivant et travaillant à Paris. Elle est diplômée d'un master en Histoire de l'Art et d'un master en Arts-Plastiques. http://fannyviollet.com [site consulté le 23/07/2020].

9. Ilaria Margutti est une artiste italienne née en 1971 à Modène. Elle vit et travaille à Sansepolcro. Conjointement à ses activités artistiques, (diplômée de l'Académie des Beaux-Arts de Florence en 1997), elle est professeur d'histoire de l'art. https://www.ilariamargutti.com/ [site consulté le 23/07/2020].

10. Ilaria Margutti, Mend of me, 2008, broderies au fil rouge sur toile, collage, mix-media.

11. Laura Mulvey, "Visual pleasure and narrative cinema », Oxford Journals, $\mathrm{n}^{\circ} 3$, automne 1975, p. 6-18.

12. Pierre Bourdieu, La Domination masculine, Paris, Seuil, 1998, p. 7.

13. Rebecca Harris, Untilted (body modification), 2013, dimension variable, cercle à broder, tissu imprimé, fil.

14. Rebecca Harris est une artiste britannique née en 1977. Ses principales expositions sont " Aesome Art Fair » en 2017 à Exeter, «Invisible You : the humain microbiome, Eden Projet », à Cornall en 2015. http://www.rebecca-harris.com [site consulté le 23/07/2020].

15. P. Bourdieu, op. cit. note 12, p. 45.

16. Ghada Amer est née en 1963 au Caire. En 1974, elle déménage avec ses parents en France et 10 ans plus tard, commence sa formation artistique à la Villa Arson à Nice. Elle vit entre New York et Paris. Son travail a été exposé à la Biennale de Venise, au Musée de Brooklyn et au Centre Pompidou. https://www.ghadaamer.com [site consulté le 23/07/2020].

17. Ghada Amer, Conseil de beauté du mois d'août, 1993, broderie sur tissu, $35 \times 35 \mathrm{~cm}$ env. 
18. Claude Fauque, La broderie: splendeurs, mystères et rituels d'un art universel, Genève, Aubanel, 2007, p. 205.

19. Valérie Vaubourg est née en 1970. Elle vit et travaille à Lille. Elle est agrégée en Arts Plastiques et titulaire d'un DNSEP obtenu aux Beaux-Arts d'Épinal. http://valerievaubourg.com/ [site consulté le 23/07/2020].

20. Valérie Vaubourg, Procné et Philomèle, encre rehaussée de fil rouge.

21. Des Lettres brodées, Maîtrise d'arts-plastiques, 1980-1982, broderies sur toiles assemblées sous forme de livre.

22. Madeleine Blondel, « Ce que disent les abécédaires », Point de croix, op. cit., note 3.

23. Yxta Maya Murray, « Rape Trauma, the State, and the Art of Tracey Emin », March 21, 2012, Loyola-LA, Legal Studies Paper, 2012.

24. Tracey Emin est née en 1963 à Londres. Elle vit entre l'Angleterre et la France. http:// www.traceyeminstudio.com [site consulté le 23/07/2020].

25. Everyone I Have Ever Slept With 1963-1995, 1995, broderie appliquée sur tente, 122x245×214 cm.

26. Aline Dallier-Popper, «Les travaux d'aiguille », Les Cahiers du Grif, n²12, juin 1976, p. 53, cité par Fabienne Dumont, dans «Aline Dallier-Popper, pionnière de la critique d'art féministe en France ", Critique d'art. Actualité internationale de la littérature critique sur l'art contemporain, $n^{\circ} 31,01 / 04 / 2008$.

\section{RÉSUMÉS}

Longtemps reléguée au rang de pratique occupationnelle, la broderie est de plus en plus plébiscitée par les artistes. Quittant son caractère décoratif, elle est qualifiée de "subversive " lorsque mise au service d'un discours féminin dénonçant la domination masculine. La couleur rouge, couleur de la violence et du sang, traditionnellement utilisée dans les abécédaires classiques et la pratique du marquage du trousseau, a été interrogée par de nombreuses artistes. La violence systémique, physique ou morale, faite aux femmes, est dénoncée par les artistes à grand recours de fil rouge. La broderie subversive contemporaine se drape d'un pouvoir discursif et permet l'écriture d'une histoire des femmes par les femmes, de l'émergence d'un matrimoine, d'un discours œuvrant à la visibilité des femmes dans la société et les institutions.

Long relegated to the rank of being a pastime, embroidery is becoming increasingly popular with artists. Forsaking its decorative nature, it is described as being "subversive" when it is used for feminist purposes that denounce male domination. Red, the colour of violence and blood, is traditionally used in classic $\mathrm{ABC}$ and the embroidered initials in the marking of a trousseau, and it has been questioned by many artists. Systemic, physical or moral violence against women is denounced by artists with a great deal of red thread. Contemporary subversive embroidery drapes itself with a discursive power and allows women to write a history of women, the emergence of female heritage, and a discourse with the goal of raising the visibility of women in society and institutions. 


\section{INDEX}

Keywords : Subversive embroidery, feminism, female heritage, herstory, textile art, gender identities, gender studies, women artists, contemporary art

Mots-clés : broderie subversive, féminisme, matrimoine, herstory, art textile, identités de genre, études de genre, artistes femmes, art contemporain

\section{AUTEUR}

\section{LÉONIE LAUVAUX}

Léonie Lauvaux a soutenu en 2018 une thèse sur l'appropriation par les plasticiennes de la pornographie mainstream par la broderie subversive. Ses thèmes de recherche sont axés sur les questions de genre, les sexualités, la maternité et le matrimoine. Parallèlement à son travail de recherche, elle développe une pratique artistique « filaire » (broderie, tricot, crochet), expose et anime des ateliers.

In 2018, Léonie Lauvaux wrote a thesis on the appropriation of mainstream pornography through subversive embroidery by visual artists. Her research focuses on gender issues, sexuality, motherhood and female heritage. Alongside her research, she engages in a "threaded" artistic practice (embroidery, knitting, crocheting), as well as exhibiting and conducting workshops. 